\title{
Criminologie
}

\section{Réparer le cercle : la responsabilisation de l'Autochtone alcoolique}

\section{Alexandra Pronovost}

Volume 42, numéro 2, automne-hiver 2009

Régulations sociopénales et peuples autochtones

URI : https://id.erudit.org/iderudit/038598ar

DOI : https://doi.org/10.7202/038598ar

Aller au sommaire du numéro

\section{Éditeur(s)}

Les Presses de l'Université de Montréal

ISSN

0316-0041 (imprimé)

1492-1367 (numérique)

Découvrir la revue

Citer cet article

Pronovost, A. (2009). Réparer le cercle : la responsabilisation de l'Autochtone alcoolique. Criminologie, 42(2), 31-51. https://doi.org/10.7202/038598ar

\section{Résumé de l'article}

Le présent article fournit une réflexion critique de la relation entre l'alcool et les Autochtones à partir d'une revue holistique des travaux produits dans le champ des sciences sociales et médicales. Les institutions juridiques et de la santé, ainsi que les milieux universitaires, ont participé à la construction d'une relation " problématique » entre l'alcool et les Autochtones. Or, le concept de l'alcoolisme devrait, selon nous, être abordé en tant que réponse culturelle et en tant que manifestation des troubles identitaires individuels et communautaires résultant des déstructurations profondes que l'insertion des Autochtones à l'État-nation a provoquées. Actuellement, l'État, sous pression de groupes politiques autochtones, tente de redonner de l'autonomie aux communautés autochtones tout en conservant une vision « victimisante » des Autochtones. L'autonomisation des peuples autochtones couplée à une "dévictimisation " peut inciter l'individu alcoolique à ne plus se considérer de cette manière, mais plutôt comme un adulte responsable envers lui-même et sa communauté. Afin d'accéder à l'autonomie gouvernementale, cette responsabilisation est nécessaire, car les communautés ont besoin de nouveaux modèles sains et autonomes.
Ce document est protégé par la loi sur le droit d'auteur. L'utilisation des services d’Érudit (y compris la reproduction) est assujettie à sa politique d'utilisation que vous pouvez consulter en ligne.

https://apropos.erudit.org/fr/usagers/politique-dutilisation/ 


\title{
Réparer le cercle: la responsabilisation de l'Autochtone alcoolique
}

\author{
Alexandra Pronovost ${ }^{1}$ \\ Étudiante \\ Département d'anthropologie, Université de Montréal \\ alexandra.pronovost@gmail.com
}

RÉSUMÉ - Le présent article fournit une réflexion critique de la relation entre l'alcool et les Autochtones à partir d'une revue holistique des travaux produits dans le champ des sciences sociales et médicales. Les institutions juridiques et de la santé, ainsi que les milieux universitaires, ont participé à la construction d'une relation "problématique» entre l'alcool et les Autochtones. Or, le concept de l'alcoolisme devrait, selon nous, être abordé en tant que réponse culturelle et en tant que manifestation des troubles identitaires individuels et communautaires résultant des déstructurations profondes que l'insertion des Autochtones à l'État-nation a provoquées. Actuellement, l'État, sous pression de groupes politiques autochtones, tente de redonner de l'autonomie aux communautés autochtones tout en conservant une vision «victimisante» des Autochtones. L'autonomisation des peuples autochtones couplée à une "dévictimisation» peut inciter l'individu alcoolique à ne plus se considérer de cette manière, mais plutôt comme un adulte responsable envers lui-même et sa communauté. Afin d'accéder à l'autonomie gouvernementale, cette responsabilisation est nécessaire, car les communautés ont besoin de nouveaux modèles sains et autonomes.

MOTS-CLÉS - Autochtones, alcoolisme, sciences sociales, anthropologie médicale, analyse de discours, Canada, Québec.

1. Je tiens à remercier chaleureusement Marie-Pierre Bousquet (Université de Montréal) et Mylène Jaccoud (Université de Montréal) pour leurs enseignements et conseils judicieux, ainsi que pour leur aide et leur appui dans la révision du présent article. 
They all still ran from drunks. All Indians grow up with drunks. So many drunks on the reservation, so many. But most Indians never drink. Nobody notices the sober Indians. (Sherman 1992: 29)

\section{Introduction}

Le dernier quart de siècle a été marqué par une amplification des discours politiques autochtones revendiquant «le droit à l'autodétermination, à l'autonomie, à la prise en charge de l'éducation, de la santé et bien entendu de l'économie [...] dans un contexte de lutte pour l'émancipation et la décolonisation» (Roy, 2002: 5). Les Autochtones ${ }^{2}$ sont ainsi devenus des acteurs majeurs sur la scène politique canadienne et mondiale. Toutefois, l'histoire qui précède cette émancipation, est celle d'une «domination maintenue, produite et reproduite dans le cadre des institutions [économiques], sociales et politiques de la société dominante» (Jaccoud, 1995: 94) dont la marginalisation des Autochtones est l'effet direct. C'est dans cette perspective que la relation entre l'alcool et les Autochtones doit être comprise. L'alcoolisme constitue la manifestation des troubles identitaires individuels et communautaires engendrés par les déstructurations profondes que ces communautés ${ }^{3}$ ont vécues dans le processus d'insertion à l'État-nation, notamment par leur «séquestration» dans les réserves fédérales, ainsi que par l'anémie économique qui en découle. Il doit aussi être davantage conçu comme un ensemble de comportements appris, plutôt qu'une prédisposition génétique propre à l'Autochtone. Cette définition inclut les abus d'alcool et les pratiques de grandes beuveries, c'est-à-dire toutes les formes de consommation considérées comme problématiques tant sur le plan individuel que communautaire. La manière de définir l'alcoolisme et ses

2. Comme Roy (2002: 1) le souligne, il est important de définir le plus justement possible les personnes qui se reconnaissent comme faisant partie des peuples qui vivaient en Amérique avant l'arrivée des Européens. Dans les milieux autochtones, l'expression «peuples des Premières Nations» est de mise, mais la lourdeur d'une telle formulation nous amène à employer le terme "Autochtone», qui prendra la majuscule s'il est employé en tant que nom. Soulignons toutefois que ce vocable, simpliste et généralisant, ne correspond pas à la complexité vécue et nous ne voulons en rien réduire la diversité des cultures autochtones multiples.

3. Tout au long de cet article, le terme communauté sera employé et nous adoptons la définition qu'en fait Roy $(2002: 7)$ : «Nous appréhendons la communauté autochtone non pas comme un tout homogène, mais plutôt comme un regroupement d'acteurs sociaux se reconnaissant, bien sûr, dans une collectivité, mais appartenant également à des "groupes d'intérêts" relativement distincts.» 
causes influence indéniablement les solutions envisagées pour y remédier et nous verrons que les points de vue diffèrent entre les acteurs concernés et les décideurs politiques. À travers la lourde structure bureaucratique et les liens de dépendance engendrés par le paternalisme étatique, les Autochtones tentent de développer des approches originales de guérison car les problèmes sociaux et la violence résultant du haut taux d'alcoolisme dans les réserves nuisent au processus d'accès à l'autonomie.

Cette réflexion critique s'effectue à partir d'une recension des écrits dans le domaine des sciences sociales et médicales. Nous nous inspirons également de nos expériences personnelles avec des collègues de travail et des concitoyens issus de communautés autochtones de l'Ouest et de l'Est canadien. L'Autochtone aux prises avec des problèmes d'alcool, ainsi que toute sa communauté, sont à la fois stigmatisés et sujets à la victimisation par l'opinion publique et gouvernementale, et ce, bien que les problèmes reliés à l'alcool ne soient pas de la même ampleur d'une réserve à l'autre et d'un individu à l'autre. Notre perspective est donc la suivante: l'individu alcoolique ne devrait plus être considéré comme une victime. Les ressources et les énergies ne devraient-elles pas être centrées sur les individus sobres ou responsables dans leurs communautés afin de promouvoir de nouveaux modèles et de nouvelles formes de contrôle social? En misant sur la prévention plutôt que sur le traitement symptomatique, sur l'intégration des savoirs autochtones et sur une prise en charge par les individus et les communautés affectées, des changements réels ne pourraient-ils pas être envisagés? Bien que plusieurs systèmes de valeurs autochtones soient fondés sur «la capacité de l'individu à être autonome, à se prendre en charge et à s'occuper de sa famille pour ne pas être un poids pour le reste du groupe» (Bousquet, 2005 : 159), la responsabilisation de l'alcoolique à travers une prise de conscience de ses maux, outre les difficultés personnelles liées à une telle démarche, est un processus qui se heurte à de nombreux obstacles structurels et normatifs, tant à l'intérieur qu'à l'extérieur des réserves autochtones, obstacles que nous explorerons dans cet article. 


\section{La prise en charge gouvernementale de l'Autochtone: un renforcement du stéréotype d'infériorité et une déresponsabilisation de l'Autochtone délinquant}

La construction de l'État-nation canadien s'est effectuée par une politique de ségrégation avec un désir avoué d'enrayer toute différence culturelle et d'assimiler l'Autochtone dans la société canadienne. L'inclusion des Autochtones s'est poursuivie par leur mise en réserve, étape primaire de l'émergence de la marginalité «où le mythe de l'infériorité des Autochtones est institutionnalisé et légalisé» (Jaccoud, 1995: 96). Protéger, civiliser et assimiler; la mission des politiques étatiques historiquement mises en place pour gérer la question autochtone au Canada ne pourrait être mieux résumée. «On voulait encourager les Indiens qui le méritaient à devenir des citoyens à part entière, mettant un terme à leur statut de mineur et à la tutelle du gouvernement à leur égard» (Dupuis, 1991: 43). S'ils étaient, au temps des premiers contacts, des alliés valorisés, le paradigme s'est déplacé vers une prise en charge gouvernementale d'individus devenus des sujets puis des pupilles de l'État. Il sera désormais attribué un double statut aux Autochtones; soit celui de mineurs en plus de celui d'individus majeurs avec le droit de vote; les Inuits depuis 1950 et les Amérindiens (au sens légal, les Indiens) depuis 1960 (Dickason, 1992). Par l'enchâssement de la Charte canadienne des droits et libertés en 1982 et la réforme constitutionnelle qui suivra peu après, le gouvernement, non sans la pression de groupes politiques autochtones, reconnaîtra et affirmera certains droits ancestraux et droits issus des traités, dont le droit à l'autonomie gouvernementale. Afin de le rendre plus éthique et acceptable, le terme pupille a été remplacé par celui de fiduciaire pour caractériser juridiquement les Autochtones. Loin de résoudre les problèmes de dépendance, ce changement maintient le statu quo : à travers le ministère des Affaires indiennes et du Nord Canada, le gouvernement conserve la responsabilité de gouverner et de gérer les Premières Nations afin de subvenir aux besoins de ces dernières. L'existence, mais surtout la grande visibilité des problèmes médicaux et judiciaires des Autochtones ont renforcé ce stéréotype d'infériorité et par le fait même légitimé leur prise en charge par l'État. De plus, la dépendance croissante des Autochtones envers l'État a résulté en l'adoption d'une habitude à «confier» la gestion de leurs désordres à ses institutions (Jaccoud, 1999). 


\section{L'institution de la santé et la médicalisation de l'alcoolisme}

Les problèmes de santé reliés à l'abus d'alcool sont très visibles et coûteux. Au sein de la population mondiale, ils représentent $4 \%$ du fardeau médical, suivant de près ceux reliés au tabagisme (Delany et al., 2008). L'alcoolisme, considéré comme une forme de dépendance biologique ou une forme de désordre mental, est un problème de santé défini et pris en charge par l'institution de la santé. Il est plus fréquemment observé au sein des réserves autochtones que dans le reste de la population canadienne (Bousquet, 2005). La souffrance sociale qu'est l'abus d'alcool est responsable de nombreuses morts par accident et meurtres, et entraîne un ensemble d'autres pathologies sociales telles que la toxicomanie, le suicide, la négligence parentale et la violence conjugale (Leroux, 1995; Bousquet, 2005; Roy, 2005). Les services de soins de la santé sur les réserves demeurent sous la responsabilité du gouvernement fédéral, malgré qu'un processus graduel de prise en charge des soins de santé par les communautés autochtones soit en cours depuis ces dernières années. Dans la perspective de l'anthropologie médicale critique, la maladie est considérée comme «l'expression de phénomènes sociaux, économiques, politiques et culturels qui transcendent tous les aspects de la vie humaine» (Roy, 2002: 15). De plus, la santé est un concept qui se définit différemment selon la culture ou le groupe d'appartenance. Si dans plusieurs systèmes de valeurs autochtones, la santé est «un état de bien-être à la fois psychologique et social qu'engendre et entretient, chez l'individu comme dans le groupe sociétal dont il fait partie, une démarche globale fondée sur l'harmonie, le respect mutuel et la fidélité à des valeurs essentielles partagées» (Petawabano et al., 1994), la biomédecine définit plutôt la santé par l'absence de la maladie, une dimension qu'elle réduit à ses manifestations biologiques (Roy, 2002: 16). Le système de santé actuel est hégémonique car il impose ses explications et ses prescriptions aux milieux autochtones, comme populaires, sans regard holistique sur l'individu et les facteurs sociaux qui l'affectent. Le concept de «biopouvoir», développé par Foucault (1975), nous enseigne que malgré la neutralité affichée des milieux de la santé, ceux-ci sont également une forme d'intervention politique. «Les centres de santé mis en place dans les communautés autochtones étaient et sont toujours des systèmes efficaces et fortement structurés exerçant une surveillance, appliquant 
une discipline et déterminant des critères de normalité et de déviance» (Roy, 2002 : 25-26). En effet, l'alcoolisme étant largement médicalisé, les professionnels de la santé se sentent légitimés de recommander et de promouvoir des mesures de contrôle en milieu autochtone. De surcroît, au fil de l'histoire, s'est bâti un préjugé voulant que l'Autochtone ne puisse supporter l'alcool aussi bien que les autres citoyens canadiens. Ce préjugé, en plus d'être enraciné dans la population allochtone, est fortement intériorisé parmi les populations autochtones elles-mêmes (May, 1994 ; Jaccoud, 1995 ; Roy 2005). L'intégration d'un tel construit a pour effet de déresponsabiliser l'individu face à sa consommation, justifiant du même coup l'intervention de l'État par le biais de ses programmes de santé publique. Toutefois, tel que la Commission royale sur les peuples autochtones le souligna, «le maintien de l'approche actuelle [jugé trop fragmentaire par les Autochtones] en matière de services ne fait que perpétuer le mauvais état de santé et les problèmes sociaux des Autochtones» (Gouvernement du Canada, 1996: 252).

\section{L'institution judiciaire et la victimisation de l'Autochtone}

Le système législatif est étroitement lié aux processus identitaires des Autochtones. C'est sous la Loi sur les Indiens, adoptée en 1876, que l'on définit qui est Indien et «il n'y a aucune autre catégorie de la population canadienne dont le sort dépende autant d'un dispositif constitutionnel» (Simard, 2003 : 94). La réglementation de la consommation d'alcool a également fait partie de ce processus identitaire dès les premiers contacts. Déjà au XvII ${ }^{\mathrm{e}}$ siècle, les autorités civiles de la Nouvelle-France considéraient la consommation d'alcool des Autochtones comme une activité déviante et responsable de leur criminalité. Aux lois françaises s'ajoutait également la pression des groupes religieux. Les jésuites, présents à cette époque, influencèrent l'adoption d'ordonnances prohibant la vente d'alcool aux Autochtones. Malgré ces ordonnances, la commission d'actes sous l'influence de l'alcool constituait une circonstance atténuante pour les Autochtones reconnus coupables d'homicides. Ils étaient d'ailleurs libérés s'il était prouvé qu'ils étaient sous l'influence de l'alcool lors de la commission de l'acte délictueux (Dickinson, 1993). L'abus d'alcool semble avoir été répandu au XviII ${ }^{e}$ et XIX ${ }^{e}$ siècles tant chez les colons que chez les Autochtones, mais ce sont ces derniers qui furent l'objet des réglementations les plus sévères. Jusqu'en 1985, l'accès, la 
possession et la consommation d'alcool sont prohibés pour les Autochtones, alors considérés comme des mineurs (Dupuis, 1991). Ces interdictions vont de pair avec les politiques assimilatrices de l'époque, car la seule manière de consommer légalement de l'alcool est de s'affranchir de son statut d'Autochtone pour embrasser exclusivement celui de citoyen canadien, sous réserve de démontrer des vertus de sobriété (Campbell, 2008). Par cette discrimination juridique, le statut d'infériorité de l'Autochtone est renforcé puisque la loi l'empêche de boire comme les autres. Qui plus est, le système de justice déresponsabilise l'Autochtone et culpabilise plutôt le contrebandier (bootlegger), c'està-dire l'Eurocanadien qui a illégalement vendu ou échangé de l'alcool avec lui. C'est pourquoi les Autochtones rencontrés en état d'ébriété autour des postes de traite ou des villes étaient interrogés afin qu'ils divulguent le nom de leurs fournisseurs et que ceux-ci soient pénalisés, voire excommuniés (Dickinson, 1993). L'impunité dont jouissaient les Autochtones en état d'ébriété devant la loi entraîna plusieurs effets pervers; depuis l'introduction des boissons alcooliques, les Autochtones se sont considérés comme innocents de tout crime commis sous leur influence (Dickinson, 1993). Dans le cadre de leurs revendications du droit à l'égalité, l'accès légal à la boisson alcoolisée a constitué l'une des luttes menées par des organisations autochtones du Canada (Campbell, 2008 : 112). Le pouvoir de gérer la libre distribution et l'accès à l'alcool a progressivement été confié aux conseils de bande à partir des années 1980. Par la reconnaissance des garanties offertes par la Charte canddienne des droits et libertés, la prohibition était désormais considérée comme une forme directe de discrimination.

Aujourd'hui, selon les circonstances, l'alcool peut être envisagé comme un facteur aggravant ou atténuant le délit dans les pratiques sentencielles des juges. Certains acteurs du milieu de la justice, jugeant que le système actuel n'a pas été mis en place pour répondre à la réalité particulière des Autochtones (Dupuis, 1991 : 57), trouvent justifiable de questionner la présumée universalité de la loi et de chercher à l'ethniciser (Adjin-Tettey, 2007). Par exemple, on cherchera dans les différences culturelles des Autochtones une explication de leur surreprésentation pénale $^{4}$. Le système de justice qui, par le passé, culpabilisait le «Blanc» pour l'apport néfaste de l'alcool à l'Autochtone, culpabilise maintenant

4. S'ils représentent $3 \%$ de la population canadienne, les Autochtones comptent pour $20 \%$ de la population incarcérée (Adjin-Tettey, 2007). 
la «société dominante» pour les fautes et les injustices commises envers les communautés autochtones à travers notre histoire. Comme le souligne Sioui (1987), ce sentiment de culpabilité «blanche» continue de rendre les Autochtones étrangers aux Allochtones, même qu'une des armes principales du mouvement d'émancipation et de reconnaissance identitaire autochtone, en essor depuis la fin des années 1960, est d'exploiter «la conscience coupable d'une majorité canadienne qui ne demande pas mieux que de se débarrasser du stigmate de «Blanc»» (Simard, 2003: 46). Par conséquent, le discours de victimisation est encore bien présent dans l'idée que le contrevenant autochtone est une victime du système qui n'est pas responsable des maux qui l'affligent et qui peut dès lors blâmer le gouvernement pour son propre comportement.

\section{Prise en charge normative en milieu autochtone}

Les Autochtones déclarent l'alcoolisme comme un des plus grands problèmes qui affligent leurs communautés (Landau, 1996). La plupart d'entre elles ne veulent plus que l'alcool soit associé à leur identité. Si la réserve a déjà été un lieu propice à l'abus d'alcool, elle doit être reconnue maintenant comme un milieu de vie sain (Bousquet, 2005). Plusieurs communautés consacrent des efforts énormes pour se dissocier de ces images négatives. Le traitement est l'une des voies privilégiées. Ainsi, que ce soit dans le cadre du Programme national de lutte contre l'abus d'alcool et de drogue chez les Autochtones (PNLAADA), des programmes d'Alcooliques anonymes, du mouvement pentecôtiste ou bien des guérisons spirituelles, les bienfaits de la désintoxication sont mis de l'avant par plusieurs communautés algonquines (Bousquet, 2005). Dans le cadre de ces programmes, on accorde de l'importance au fait de repositionner l'alcoolisme dans son contexte socioculturel. De plus, par la déconstruction de la relation entre l'alcool et les Autochtones, une prise de conscience de l'enracinement historique des stéréotypes associés est favorisée. Le regard diachronique sur l'émergence des problèmes est crucial et, comme le souligne si bien Ben Okri (1996: 12), un romancier du Nigeria: «If they tell themselves stories that are lies, they will suffer the future consequences of those lies. If they tell themselves stories that face their own truths, they will free their histories for future flowerings.»

Ainsi, les programmes de prévention issus des communautés sont davantage axés sur l'éducation, la rééducation et la prise en charge 
sociale et individuelle. Par divers moyens, on vise à renverser les tendances meurtrières sur les réserves où le comportement de l'alcoolique est appris et occasionne un cycle vicieux transmis de manière intergénérationnelle (Comack et Peters, 2005). Par exemple, conséquent avec sa volonté de s'autonomiser, le conseil de bande de la communauté de Nutashkuan (communauté innue de la Basse-Côte-Nord) a pris en charge les services sociaux et d'aide à l'enfance et à la famille dès 1997. On y a fondé, en 1999, le projet Usseniun, signifiant en innu «Vie nouvelle». Soucieux de retrouver un mieux-être individuel, familial et communautaire, les Autochtones et Allochtones actifs dans ce projet se sont engagés dans une réflexion où se sont conjugués savoir thérapeutique des Aînés et savoir professionnel des intervenants psychosociaux. Les cinq autres communautés de la Basse-Côte-Nord disposent aujourd'hui de projets calqués sur le projet Usseniun, mais personnalisés selon leurs caractéristiques propres. L'un des enjeux de ces projets est de modifier les perceptions. C'est ainsi que le geste de consommation abusive est maintenant compris comme une conséquence d'un processus historique et non plus comme un choix individuel à la source de tous les maux; il «est conséquemment devenu l'objet d'un processus de responsabilisation» (St-Arnaud et Bélanger, 2005: 168). En comprenant mieux le geste de consommer, les individus sont maintenant en position de force face à leurs problèmes devenus plus surmontables. L'accent est également mis sur l'importance que l'ensemble de la communauté collabore dans le processus de guérison. En effet, la prévention, c'est aussi consacrer des efforts aux individus qui n'ont pas encore adopté de comportements problématiques. L'interconnexion entre les programmes de prévention et thérapeutiques offre des perspectives encourageantes. Le traitement, lorsqu'il réussit, permet aux individus désintoxiqués de devenir des modèles de sobriété. On accorde alors de l'importance à la sobriété ou du moins à une consommation responsable de l'alcool. Il s'agit, dans le symbolique, d'une prise en charge sociale (Bousquet, 2005). Ces nouveaux modèles ont permis aux victimes de violence, de négligence parentale ou des viols perpétrés par des individus en état d'ébriété de se faire entendre. C'est le cas par exemple de la communauté anicinape (algonquine) de Kitcisakik, particulièrement touchée par l'alcoolisme (Leroux, 1995). Dans cette communauté, un puissant processus d'amélioration de la santé communautaire et de restructuration des organisations sociales a été engendré par le travail du conseil de bande et de la Société de bien-être Kitcisakik, 
fondée en 1980. Les acteurs principaux de ce processus ayant majoritairement connu un passé marqué par les beuveries et l'alcoolisme sont d'abord devenus sobres pour gagner la confiance des membres de leur communauté, étape nécessaire pour ensuite entreprendre des actions et provoquer des changements visant le bien-être de ces derniers.

\section{Les obstacles à l'essor de nouvelles normes}

En dépit de la volonté de prise en charge des institutions de la santé exprimée par plusieurs communautés autochtones, et en dépit du succès des projets de prévention et d'intervention émanant de ces milieux, les gouvernements continuent d'analyser le phénomène de l'alcoolisme en milieu autochtone de manière à justifier le déploiement de ressources et d'interventions axées sur le traitement. Une conséquence immédiate des orientations du système socio-sanitaire dans son ensemble est la préoccupation axée sur les soins davantage que sur la prévention (Simard, 2005: 312). De plus, les interventions s'effectuent au sein d'institutions créées dans un paradigme colonialiste et paternaliste. Le path dependency est cette «logique institutionnelle intrinsèque qui soustend continuellement la gestion étatique du dossier autochtone [qui] ne s'est guère modifiée avec le temps: les peuples autochtones au Canada demeurent des objets de politiques et représentent aux yeux du législateur une question à résoudre, un problème à régler [...]» (Ladner et Orsini, 2004: 79). Qui plus est, parce que l'action gouvernementale intervient là où des problèmes surgissent, plusieurs acteurs sociaux des milieux autochtones emploient la rhétorique du corps malade pour attirer l'attention gouvernementale et les ressources qui l'accompagnent, contribuant à fixer une image inférieure et victimisante d'eux-mêmes: «increasingly the Indian must define himself as sick in order to get federal monies»(Levy, 1976, in Piron, 1994: 124). Ainsi, malgré l'injection d'importants investissements humains, scientifiques et financiers, peu de progrès notables sont observés dans la manière dont les services de santé sont rendus aux individus affectés (Delany et al., 2008). Plusieurs considérations peuvent nous aider à comprendre l'inefficacité de l'intervention gouvernementale.

Premièrement, les milieux autochtones ne sont pas hégémoniques; les opinions divergent concernant le pouvoir politique, divergence qui prend la forme de luttes intéressées ou idéologiques. Prenons par exemple, sur le plan national, l'opposition entre l'Assemblée des 
Premières Nations du Québec et du Labrador et l'Association femmes autochtones. Les femmes autochtones se battent pour que l'égalité des sexes, principe fondamental de la Charte des droits humains s'applique au domaine autochtone, mais les chefs craignent «d'affaiblir les prérogatives attachées au statut, par dilution démographique dans le premier cas, par une compétence supérieure, dans le second» (Simard, 2003: 95). Sur le plan local, les conseils de bande, créés et imposés aux communautés autochtones par la Loi sur les Indiens de 1876, sont considérés par plusieurs comme une simple continuité du ministère des Affaires indiennes et du Nord Canada. L'assise politique de ces entités politiques locales est donc souvent remise en cause. Ce fut le cas en 1992, chez les Innus de Malioténam; en 1996, chez les Algonquins de Lac-Barrière; en 1998, chez les Micmacs de Restigouche (Morissette, 2007: 127). Deuxièmement, l'homogénéité des niveaux de vie est également un mythe car il existe une classe moyenne et une élite politique autochtone. La conséquence de cette différenciation sociale est que les débats concernant des choix collectifs n'ont plus lieu dans les communautés mais autour de tables rondes officielles et que ceux-ci portent davantage sur «les modalités juridico-administratives du patronage que l'État se doit d'exercer à l'endroit de ses pupilles [fiduciaires] que sur les possibles et les tâches à effectuer par les Autochtones en tant que citoyens» (Simard, 2003 : 33). Troisièmement, l'uniformité des politiques publiques appliquées à l'ensemble des réserves autochtones de la province, voire du territoire canadien, ne tient pas compte des spécificités et des différences culturelles qui existent entre les diverses Premières Nations du pays. Cette tendance à nier les spécificités locales et régionales au profit d'un mieux-être généralisé est issue de la mondialisation, dont Roy (2002: 9) considère que les peuples autochtones $\mathrm{du}$ monde sont les premières victimes. Quatrièmement, l'échec des interventions étatiques s'explique également par la stigmatisation que reçoit l'ensemble des membres d'une communauté considérée comme problématique. En effet, sans regard critique porté sur les statistiques concernant la délinquance des Autochtones, le comportement problématique d'une minorité d'individus, rendu visible par les consultations, les cures de désintoxication et les incarcérations répétées, peut porter ombrage à l'ensemble de la communauté. Une mauvaise interprétation des statistiques comporte alors les risques de nous induire en erreur en plus de camoufler l'extrême variabilité qui existe entre les conditions de vie des résidents d'une même réserve. Cinquièmement, comme 
l'explique Laprairie (1989: 146), «la race comme variable discrète sous-tend des politiques et des programmes, à l'exclusion d'autres facteurs tels que la pauvreté, la ruralité et/ou l'isolement géographique», entraînant ainsi une surreprésentation «ethnique ${ }^{5}$ » du problème de l'alcoolisme, ce qui contribue à la stigmatisation unanime des Autochtones. Comme Kleinman (1995) le soutient, les forces sociales, économiques, politiques et institutionnelles peuvent infliger des souffrances sociales à l'expérience humaine. En dernier lieu, les études comparent très rarement la situation vécue par les autochtones avec celle vécue par d'autres minorités colonisées dans le monde. Déjà au début du $\mathrm{xx}^{\mathrm{e}}$ siècle, Durkheim (1897) définit l'anomie comme étant un isolement social et une absence de normes culturelles et de contraintes sociales intériorisées découlant de la déstructuration des valeurs familiales par l'urbanisation accélérée des milieux. De cette manière, Wilson (1996) explique les pathologies anomiques qui accablent les ghettos noirs (dont l'alcoolisme) par l'écart qui existe entre l'émancipation des désirs et les possibilités effectives de les réaliser, à cause de ce qu'il appelle «la disparition du travail» (When work disappears). Les changements rapides et le manque de travail sont des réalités structurelles non négligeables en milieu autochtone; ces réalités devraient teinter davantage les discours interventionnistes.

\section{Résister à la victimisation dans des milieux déstructurés}

Dans plusieurs réserves autochtones, les pratiques de consommation d'alcool sont devenues un «fait indien» et s'inscrivent dans un puissant code identitaire dans lequel un nombre significatif d'Autochtones se reconnaissent et auquel ils se conforment (Roy, 2005). Constatant que l'abus de l'alcool est ancré dans les mœurs au point de devenir normal, Bousquet (2005), reprenant l'expression de Tremblay (1995), emploie le concept de «sous-culture de l'alcoolisme» pour parler des comportements et des normes qui tolèrent et encouragent la consommation excessive chez les Algonquins. Bousquet (2005) observe que l'individu qui prend la décision de se désintoxiquer se trouve marginalisé au sein de sa propre communauté en raison de sa dérogation aux normes domi-

5. Nous considérons l'ethnicité comme le produit d'un rapport social entre groupes disposant de ressources et de pouvoirs différenciés (Jaccoud, 2003). L'ethnicité est une identification subjective et non une affaire de gênes. 
nantes. Dans son analyse de l'épidémie de diabète en milieu innu, Roy (2002) en arrive aux mêmes conclusions. Cela n'est pas unique aux Autochtones, chez les Dockers du Havre, l'homme devenu sobre est également désolidarisé de son ancien cercle d'amis buveurs (Roy, 2005). Cette surconsommation tolérée est trop souvent expliquée de manière simpliste du fait que la culture autochtone est appréhendée comme «une entité profondément enracinée dans une ancestralité, une tradition s'inscrivant en faux avec la modernité» (Roy, 2002: 21). Les spécialistes expliqueront le haut taux de maladie ou de déviance en milieu autochtone par deux types de facteurs: soit les Autochtones ont été contaminés par la modernité et sont donc vus comme des victimes passives et impuissantes, soit ils refusent et résistent à la modernité et à ses apports bénéfiques telle la technologie biomédicale. Les échecs répétés de l'intervention gouvernementale en matière de santé nécessitent toutefois une réflexion plus profonde écartant ces dichotomies réductrices de colonisé/colonisateur ou de tradition/modernité. Comme le rappelle Dupuis (1991), afin d'affirmer leur autonomie politique et d'assurer leur survie culturelle, plusieurs Autochtones affichent leurs réticences envers les institutions et les programmes d'intervention émanant des gouvernements, qu'ils soient considérés «comme un mal nécessaire, comme une ingérence dans leurs affaires [ou] comme une tentative supplémentaire de les assimiler» (1991: 66) ou comme une forme de colonialisme interne (Roy, 2005). La non-adhésion aux prescriptions des milieux de la santé est alors analysée comme une résistance qui est «au quotidien, l'affirmation de la spécificité, de la distinction, de l'unicité, de l'identité, de l'autonomie dans l'espace de l'exercice du biopouvoir» (Roy, 2002: 27). Cette réaction s'observe également dans d'autres populations, dont les populations des villes de l'Abitibi, où Bibeau et Corin (1995) ont observé que, à la suite des déstructurations encourues par la baisse des activités forestières, les comportements problématiques les plus répandus étaient marqués par l'excès (alcool ou violence), mais que leur portée était minimisée dans le discours des informateurs afin de les présenter comme étant en continuité avec leur mode de vie et leur conception de la personne valorisée. Cette attitude était adoptée en tant que stratégie défensive pour protéger un sentiment collectif de survie et de maîtrise sur sa vie. Ainsi, ces groupes d'individus déploient des efforts pour se protéger contre les inégalités, l'exploitation, l'assimilation et la manipulation, et l'individu est un acteur dynamique et créatif et non une victime passive. 
Toutefois, force est d'admettre que les mécanismes de contrôles sociaux traditionnels, telle la famille, sont en voie de recomposition depuis quelques années (Bousquet, 2005). Les discours, tant autochtones qu'allochtones, portant notamment sur les systèmes traditionnels de règlements de conflits, élèvent la communauté au rang de l'élément le plus important des cultures autochtones. En ce qui a trait aux Amérindiens, la nature élargie des familles et la composition des groupes de chasse ont favorisé l'essor d'un esprit collectif fort, mais les relations de contiguiité prolongée propre à la vie en réserve demeurent une réalité récente pour ces derniers. Bousquet (2005) rappelle qu'il importe de demeurer fidèle à sa communauté, mais il est surtout important de rester fidèle à sa famille. La famille était traditionnellement une institution d'apprentissage et de contrôle des normes de comportement. Ces structures normatives étaient alors soutenues par des réseaux de solidarité découlant des modes de vie du semi-nomadisme (Leroux, 1995). À cette époque, pour subvenir à leurs besoins, deux ou trois familles, réunies en groupes de chasse, devaient se déplacer sur leurs territoires de chasse (Gélinas, 2003 : 204). Tout convergeait pour autonomiser la famille comme unité économique quasi indépendante (Simard, 2003 : 159). Par conséquent, le droit de circuler librement et l'autonomie individuelle étaient des valeurs cruciales qui demeurent encore importantes de nos jours. Or, la consommation excessive d'alcool est une action qui entrave la liberté et l'autonomie individuelle et mène très souvent à des comportements destructeurs envers des membres de la communauté. Comme Dickinson (1993) le souligne, par le passé, les Autochtones ont attribué à l'ébriété une excuse aux actes commis et ils pouvaient donc transgresser les règles communautaires sans craindre des sanctions individuelles. De son côté, Leroux (1995) laisse entendre que cette mentalité persiste dans plusieurs communautés autochtones, car le démantèlement historique des réseaux de solidarité traditionnels, causé par le bouleversement récent des modes de vie, a profondément transformé les systèmes de conduite, notamment par l'étiolement des valeurs. Par un procédé de nostalgie, où les « riches dimensions perdues de la vie sociale trouvent à se reproduire sous de nouvelles formes socioculturelles» (Simard, 2003: 75), les valeurs se modifient et c'est dans ce sens que, dans certaines communautés, il est plus aisé de se bâtir un réseau de solidarité pour aller boire que pour cesser de boire (Roy, 2002; Bousquet, 2005). C'est également ainsi que persiste l'éthique de la non-intervention, où il est mal vu de se mêler des affaires des 
autres (Whitehead et Hayes, 1998). Par conséquent, on ferme les yeux et l'on hésite à dénoncer les abus qui ont lieu dans une autre famille, voire dans notre propre famille. Cette réalité n'est pas propre aux Autochtones car l'importance de la famille, comme cadre et limite du soutien social, est également observée par Bibeau et Corin (1995: 56): «autant le soutien passe par la famille, autant on n'intervient pas dans l'espace familial de l'autre et on respecte la sphère du privé ». De plus, selon Leroux (1995), la loi du silence pèse sur les victimes d'actes violents où la préservation du réseau social familial prédomine sur la souffrance de ces derniers. Des règles tacites, qui vont à l'encontre de certains droits et libertés prévus dans les chartes, s'imposent de la sorte dans les communautés. Certains individus, notamment les femmes et les enfants, se retrouvent par conséquent sans protection par rapport aux abus de pouvoir et au contrôle normatif exercé par la minorité d'individus problématiques. Au nom de la revitalisation des traditions ou de la préservation de la culture, la liberté individuelle et les droits qui en découlent se trouvent subordonnés aux intérêts communautaires censément plus importants (Adjin-Tettey, 2007). Puisque le rôle régulateur et normatif de la famille s'est effrité, il devient important qu'une autre institution se substitue à cette fonction et qu'une forme de protection des victimes d'actes violents soit mise en place.

\section{La judiciarisation de la violence sur les réserves}

La loi du silence neutralise la judiciarisation de la violence sur les réserves. De plus, le système judiciaire est connoté négativement par la représentation que s'en font plusieurs Autochtones. Cette perception peut se traduire par une méfiance accrue envers cet outil de l'État, qui ne reconnaît pas leurs droits, et dont la collaboration équivaudrait à établir un pacte avec l'ennemi. Ainsi, il vaut mieux «supporter les problèmes à l'intérieur de la communauté que de dénoncer ou de contribuer à faire condamner l'un des siens par la justice non indienne» (Dupuis, 1991 : 57). Tant et aussi longtemps que de nouveaux mécanismes de contrôle ne seront pas mis en place par les communautés, l'intervention du système pénal gouvernemental ne peut être totalement rejetée, car celui-ci peut constituer la seule forme de protection des victimes d'actes violents. Depuis les années 1980, les milieux de la justice sont ouverts à l'idée d'une justice réparatrice, dont les cercles de guérison sont un exemple concret. Jaccoud (1999) avance que la guérison est incompatible avec 
l'exclusion du délinquant, car elle nécessite, comme le souligne également Leroux (1995), le rétablissement des relations sociales entre la victime, le délinquant, leurs familles respectives et la collectivité entière. Les cercles de guérison illustrent bien les divers points soulevés ici, car pour s'engager dans le cercle, le délinquant doit tout d'abord reconnâ̂tre et accepter sa responsabilité individuelle (Jaccoud, 1999). En somme, les cercles de guérison constituent une étape importante vers un transfert des pouvoirs aux collectivités autochtones et par le fait même vers une prise en charge de leur propre destinée. Aux cercles de guérison s'ajoute la pratique de cercles de sentence, un exemple tangible de la collaboration entre le système de justice gouvernementale et les communautés autochtones. Seul le juge peut légitimer cette pratique, qui consiste à faire participer la communauté au processus d'imposition des sanctions, contribuant ainsi à l'élaboration de recommandations plus adaptées aux circonstances et aux acteurs concernés par le conflit (Jaccoud, 1999). La présence des juges aux cercles ne fait pas consensus au sein de la pratique judiciaire et des communautés. D'une part, leur absence semblerait faciliter les échanges et les recommandations, d'autre part, leur présence a l'avantage de contrebalancer les distributions inégales de pouvoir. En effet, étant donné que les oppositions à la judiciarisation de la violence sont fortement influencées par la complaisance avec laquelle certains agresseurs tirent profit de la loi du silence, il est nécessaire que les victimes soient incitées à dénoncer leurs agresseurs et donc, faute d'écoute au sein de la réserve, une oreille extérieure devient nécessaire (Leroux, 1995).

\section{Conclusion}

Depuis la fin de la guerre mondiale, les peuples colonisés du monde entier sont engagés dans un processus de décolonisation. Malheureusement, l'affirmation d'une autonomie gouvernementale devient beaucoup plus difficile lorsque les peuples colonisés sont enclavés dans le territoire de leurs colonisateurs, comme c'est le cas pour les communautés autochtones du Canada. Or, «si l'expérience autochtone a dit à ceux-ci qu'ils étaient incapables de s'occuper d'eux-mêmes, la période postcoloniale ou de décolonisation est une période de contestation de l'impérialisme occidental et de ses structures coloniales et dominatrices» (Adjin-Tettey, 2007: 187). Dans cette lutte, les inégalités structurales sont les plus difficiles à surmonter, car elles sont profondément enracinées, surtout 
l'écart socioéconomique entre les Autochtones et les Allochtones, maintes fois souligné ${ }^{6}$, qui demeure la toile de fond des problèmes de la surconsommation d'alcool dans les réserves. Cet écart est attribuable aux oppressions d'un système capitaliste et des politiques colonialistes. Tout au long de leur colonisation, l'exclusion des Autochtones s'est concrétisée par le renforcement de leur différence avec le monde des Blancs, notamment dans leurs habitudes de consommation. Aujourd'hui, cette rhétorique se voit réappropriée et réutilisée par les Autochtones comme «instrument politique du changement social» (Jaccoud, 1994: 97). Se définir comme exclus sur la base d'une différence peut, comme le souligne Adjin-Tettey (2007), constituer une arme de changement social, mais ne doit pas cautionner la violence. Force est d'admettre que malgré les visées assimilationnistes des politiques gouvernementales, l'identité autochtone s'est maintenue à travers toute l'histoire et tout l'espace du Canada (Dickason, 1992). Comme les Canadiens français à la Révolution tranquille, il est intéressant de voir la crise identitaire se cristalliser en organisations politiques créatrices de forces de changement qui démontrent les capacités indéniables de prise en charge dont disposent les milieux autochtones et que cette voie offre une meilleure santé aux populations. L'étude comparative de Chandler et Lalonde (2004) est très convaincante à cet égard car elle démontre que là où les communautés ont acquis des formes d'autonomie et de contrôle, les taux de suicide sont considérablement plus bas, voire nuls, comparativement aux communautés marquées par une plus grande dépendance politique. Les communautés autochtones ont une population composée d'un très grand nombre de jeunes de moins de 25 ans. Cette génération, née dans une réserve (Morissette, 2007), a accès aux savoirs à la fois traditionnels et modernes, ce qui lui permet d'innover et de façonner de nouvelles facettes culturelles et identitaires, à condition d'évoluer dans un environnement sain et créatif;

Fermez les cabarets, ouvrez les écoles. L'ivrognerie dévore le peuple (Zola, L'assommoir).

6. Robbins, 1973; Charest et Tanner, 1992; Piron, 1994; Jaccoud, 1995; Leroux, 1995; Whitehead et Hayes, 1998; Bousquet, 2005; Roy, 2005; Delany et al., 2008. 


\section{Références}

Adjin-Tettey, E. (2007). Sentencing aboriginal offenders : balancing offenders' needs, the interests of victims and society, and the decolonization of aboriginal peoples. Canadian Journal of Women and the Law, 19 (1), 170-216.

Bibeau, G., \& Corin, E. (1995). Culturaliser l'épidémiologie psychiatrique. Les systèmes de signes, de sens et d'actions en santé mentale. In F. Trudel, P. Charest \& Y. Breton (Eds.), La construction de l'anthropologie québécoise. Mélanges offerts à Marc-Adélard Tremblay (211-227). Québec: Presses de l'Université Laval.

Bibeau, G. (2000). Qui a peur des ethnies? Questions (subversives) aux politologues canadiens et québécois. In M. Elbaz \& D. Helly (Eds.), Mondialisation, citoyenneté et multiculturalisme (171-210). Québec: Les Presses de l'Université Laval.

Bousquet, M. P. (2005). La production d'un réseau de sur-parenté: histoire de l'alcool et désintoxication chez les Algonquins. Drogues, santé et société, 4 (1), 133-169.

Brassard, R. (2005). L'expérience de l'enfermement carcéral des femmes autochtones au Québec. Canadian Journal of Women and the Law, 17 (2), 311-340.

Campbell, R. (2008). Making sober citizens: The legacy of indigenous alcohol regulation in Canada, 1777-1985. Journal of Canadian Studies, 42 (1), 105126.

Chandler, M. J., \& Lalonde C. E. (1998). Cultural continuity as a hedge against suicide in Canada's First Nations. Transcultural Psychiatry, 35 (juin), 191219.

Chandler M. J., \& Lalonde C. E. (Sous presse). Cultural continuity as a moderator of suicide risk among Canada's First Nations. In L. Kirmayer \& G. Valaskakis (Eds.), The Mental Health of Canadian Aboriginal Peoples: Transformations, Identity, and Community. Vancouver : University of British Columbia Press. Consulté le 20 mars 2009, http://web.uvic.ca/ lalonde/manuscripts/2004Transformations.pdf.

Charest, P., \& Tanner, A. (1992). La reconquête du pouvoir par les Autochtones, Présentation. Antbropologie et Sociétés, 16 (3), 5-16.

Comack, E., \& Peter, T. (2005). How the criminal justice system responds to sexual assault survivors: The slippage between «responsibilization» and «blaming the victim». Women and the law, 17 (2), 283-309.

Coyhis, D., \& Simonelli, R. (2008). The native american healing experience. Substance Use or Misuse, 43 (12), 1927-1949.

Dailey, R. C. (1968). The role of alcohol among north american indian tribes as reported in the jesuit relations. Anthropologica, 10 (1), 45-57.

Delany, P., Shields, J., Willenburg, M., \& Huebner, R. B. (2008). Expanding the role of health services research as a tool to reduce the public health burden of alcohol use disorder. Substance Use and Misuse, 43 (12), 17291746.

Dickason, O. P. (1992). Canada's First Nations: A History of Founding Peoples from Earliest Times. Norman: University of Oklahoma Press. 
Dickinson, J. (1993). «C'est l'eau-de-vie qui a commis ce meurtre». Alcool et criminalité amérindienne à Montréal sous le régime français. Études canddiennes, 35, 83-94.

Dupuis, R. (1991). La question indienne au Canada. Québec: Les éditions du Boréal.

Durkheim, É. (1897). Le suicide. Étude de sociologie. Paris: Les Presses universitaires de France.

French, L. A. (2004). Alcohol and other drug addictions among native americans: The movement toward tribal-centric treatment programs. Alcobolism Treatment Quaterly, 22 (1), 81-91.

Foucault, M. (1975). Surveiller et punir: naissance de la prison. Paris: Gallimard.

Gélinas, C. (2003). Entre l'assomoir et le godendart. Les Atikamekw et la conquête du Moyen-Nord québécois 1870-1940. Silleray: Septentrion.

Gouvernement du Canada (1996). Rapport de la commission royale sur les peuples autochtones: Vers un ressourcement, vol. 3. Commission royale sur les peuples autochtones. Ottawa: Ministère des Approvisionnements et Services du Canada.

Harold, J. (2000). Alcohol, Freedom and Responsibility. Journal of Health Politics, Policy and Law, 25 (4), 760-769.

Jaccoud, M. (1995). L'exclusion sociale et les Autochtones. Lien social et politiques, 34, 93-100.

Jaccoud, M. (1999). Les cercles de guérison et les cercles de sentence autochtones au Canada. Criminologie, 32 (1), 79-105.

Jaccoud, M. (2003). Le construit de l'ethnicité en criminologie. Criminologie, $36(2), 3-7$.

Kleinman, A. (1995). Writing at the Margin: Discourse between Antbropology and Medecin. Berkeley/Los Angeles: University of California Press.

Kleinman, A., \& Kleinman, J. (1996). The appeal of experience; the dismay of images: Cultural appropriations of suffering in our times. Daedalus, 125 (1), $1-23$.

Landau, T. (1996). Policing and security in four remote aboriginal communities: a challenge to coercive models of police work. Revue Canadienne de Criminologie, 38 (1), 1-32.

LaPrairie, C. (1989). La justice pénale chez les Autochtones du Canada: principes et pratiques. Anthropologie et Sociétés, 13 (1), 143-154.

Larose, F., Bourque, J., Terrisse, B., \& Kurtness, J. (2001). La résilience scolaire comme indice d'acculturation chez les autochtones: bilan de recherches en milieux innus. Revue des sciences de l'éducation, 27 (1), 151-180.

Leroux, J. (1995). La métamorphose du pacte dans une communauté algonquine. Recherches amérindiennes au Québec, 25 (1), 51-69.

Levy, J. E. (1976). Introduction. In J. Leland (1976). Firewater myths: North American Indian Drinking and alcobol addiction. New Brunswick (NJ): Rutgers Center of Alcohol Studies. 
May, P. A. (1994). The epidemiology of alcohol abuse among american indians: The mythical and real properties. American Indian culture and research journal, 18 (2), 121-143.

Morissette, A. (2007). Composer avec un système imposé: La tradition et le conseil de bande à Manawan. Recherches amérindiennes du Québec, 37 (2-3), 127-138.

Norvez, A. (1993). Alcoolisation et Alcoolisme au Canada. Études canadiennes, 35, 73-81.

Okri, B. (1996). Dangerous Love. Phoenix: Phoenix House Publishing.

Petawabano, B. H., Gourdeau, E., Jourdain, F., Palliser-Tugulak, A., \& Cossette, J. (1994). La santé mentale et les autochtones du Québec. Montréal: Éditions Gaëtan Morin.

Piron, F. (1994). Production de savoir et effets de pouvoir. Le cas de la délinquance des Autochtones au Canada. Anthropologie et Société, 18 (1), $107-$ 132.

Robbins, R. H. (1973). Alcohol and the identity struggle: Some effects of economic change on interpersonal relations, American Antbropologist, 75 (1), 99-122.

Roy, B. (2002). Sang sucré, pouvoirs codés, médecine amère. Diabète et processus de construction identitaire: les dimensions sociopolitiques du diabète chez les Innus de Pessamit. Québec: Presses de l'Université Laval.

Roy, B. (2005). Alcool en milieu autochtone et marqueurs identitaires meurtriers. Drogues, santé et société, 4 (1), 85-128.

Simard, J-J. (2003). La réduction. L'autochtone inventé et les Amérindiens d'aujourd'bui. Sillery: Septentrion.

Sioui, G. E. (1987). Pour une autohistoire amérindienne: essai sur les fondements d'une morale sociale proprement américaine. Québec: Presses de l'Université Laval.

Torrie, J. (1989). Soûlards indiens et flics blancs. Considérations théoriques sur la récidive. Anthropologie et Sociétés, 13 (1), 126-142.

Tremblay, A. (1995). L'organisation de la santé dans une réserve montagnaise», Recherches amérindiennes au Québec, 25 (1), 21-40.

Sherman, A. (1992). The Business of Fancydancing. Brooklyn: Hanging Loose.

St-Arnaud, P., \& Bélanger, P. (2005). Co-création d'un espace-temps de guérison en territoire ancestral par et pour les membres d'une communauté autochtone au Québec. Appréciation clinique d'une approche émergente et culturellement adaptée. Drogues, santé et société, 4 (2), 141-176.

Stratton, J. (1973). Cops and Drunks: Police attitudes and actions in dealing with indian drunks. International Journal of Addictions, 8 (4), 613-621.

Westermeyer, J. (1974). The Drunken Indian: Myths and Realities. Psychiatric Annals, 4 (11), 29-36.

White, W. L. (2004). Native American Addiction: A response to French. Alcobolism Treatment Quaterly, 22 (1), 93-97.

Whitehead, P. C. \& Hayes, M. J. (1998). The insanity of alcobol: Social problems in canadian first nations communities. Toronto: Canadian Scholars' Press. 
Wilson, W. J. (1996). When work disappears. The world of the new urban poor. New York: Alfred Knopf.

ABSTRACT • Based on the scientific literature produced by social and medical sciences, this article presents a critical reflexion on the relationship between alcohol and Natives. Throughout Canadian history, the state and academia participated in the construction of this "problematic" relationship. With this knowledge, we better understand the government's misinformed intervention politics. The concept of alcoholism should be considered a cultural response. It is also a manifestation of the complex individual and community identity struggles, engendered by the inclusion of native communities in the nation state. Today, under the pressure of native political groups, the state tries to give back autonomy to native communities while maintaining a victimising view of them. "De-victimisation" would allow the alcoholic individual to consider himself a responsable adult and thus strengthen his community. To access self-gouvernance, active responsibility is a necessary and difficult first step because communities are in need of new, healty and autonomous models.

KEYWORDS - Aboriginal Peoples, Alcoholism, Social sciences, medical anthropology, discourse analysis, Canada. Quebec.

RESUMEN - Este artículo presenta una reflexión crítica de la relación entre el alcohol y los pueblos autóctonos a partir de una amplia revisión de los trabajos generados en las ciencias sociales y la medicina. Las instituciones jurídicas y de salud, al igual que el medio universitario, han participado en la construcción de una relación "problemática" entre el alcohol y las comunidades autóctonas. Sostenemos, sin embargo, que el concepto de alcoholismo debería abordarse en tanto que respuesta cultural y manifestación de los problemas de identidad individuales y comunitarios que resultan de las desestructuraciones profundas provocadas por la inserción de los pueblos autóctonos en el Estado-nación. En la actualidad, el Estado trata de restablecer la autonomía a las comunidades autóctonas, pero conserva una visión "victimizante" de estas comunidades. La autonomización de los pueblos autóctonos, junto con "desvictimización", pueden incitar al individuo alcohólico a ya no considerarse como tal, sino como un adulto responsable consigo mismo y con su comunidad. Esta responsabilización es necesaria a fin de acceder a la autonomía gubernamental, en la medida en que las comunidades necesitan nuevos modelos sanos y autónomos.

PALABRAS CLAVE - Autóctonos, alcoholismo, ciencias sociales, antropología médica, análisis de discurso, Canadá, Quebec. 